\title{
Novelty Design in Gain Flattening Filter of ASE Source Based on Fat Ultra-Long Period Fiber Grating
}

\author{
Fereshteh Mohammadi NAFCHI $^{1}$, Sharifeh SHAHI ${ }^{2 *}$, \\ Mohammad Taha SHAFFAATIFAR ${ }^{3}$, Mohammad KANANI ${ }^{2}$, and \\ Hossein NOORMOHAMMADI ${ }^{2}$
}

\author{
${ }^{1}$ Department of Electrical Engineering, Majlesi Branch, Islamic Azad University, Majlesi, Isfahan, Iran \\ ${ }^{2}$ Dental Biophotonics and Laser Research Center (DBLRS), Khorasgan (Isfahan) Branch, Islamic Azad University, \\ Arghavanieh, Isfahan, Iran \\ ${ }^{3}$ Department of Computer Engineering, Najafabad Branch, Islamic Azad University, Najafabad, Isfahan, Iran \\ *Corresponding author: Sharifeh SHAHI～E-mail: shahilaser@khuisf.ac.ir
}

\begin{abstract}
A new type of gain flattening filter for amplified spontaneous emission (ASE) source based on erbium doped fiber (EDF) is proposed and demonstrated by fabricating and writing two series ultra-long period fiber grating (ULPFG) on single mode fiber (SMF-28). The novelty method in this research is based on writing the two ULPFGs as fat gratings. The LPG is written by a simple and available arc-discharge method. The pump power based on single-pass backward pump configuration is around $100 \mathrm{~mW}$, and the average wavelength is near to $974 \mathrm{~nm}$. The gain flattening profile is obtained by $3.4( \pm 1.7) \mathrm{dB}$ ripple in the wavelength range between $1524 \mathrm{~nm}$ and $1565 \mathrm{~nm}$ with 41-nm band width.
\end{abstract}

Keywords: Amplified spontaneous emission (ASE) source; erbium-doped fiber amplifier (EDFA); gain flattening filter; long-period fiber grating (LPFG)

Citation: Fereshteh Mohammadi NAFCHI, Sharifeh SHAHI, Mohammad Taha SHAFFAATIFAR, Mohammad KANANI, and Hossein NOORMOHAMMADI, "Novelty Design in Gain Flattening Filter of ASE Source Based on Fat Ultra-Long Period Fiber Grating," Photonic Sensors, 2016, 6(3): 243-248.

\section{Introduction}

Erbium doped fiber amplifier is an essential component for increasing capacity of transmission in long-way communication systems [1]. Although these amplifiers are more useful than others, un-flatness gain spectrum of amplified spontaneous emission (ASE) source around $1532 \mathrm{~nm}$ leads to decrement in capacity and bandwidth in wavelength division multiplexing (WDM) and dense wavelength division multiplexing (DWDM) systems. Hence, the gain flattening of erbium-doped fiber amplifier (EDFA) is serious for these systems. The using of several pump configurations, such as forward or backward and single or double pass EDFA, has been proposed during years [2-4]. The gain fluctuation was obtained around $0.9 \mathrm{~dB}$ for the wavelength between $1560 \mathrm{~nm}$ and $1610 \mathrm{~nm}$ with $15-\mathrm{m}$ EDF length for the pumping with single pass forward (SPF) configuration [2]. The gain spectrum is a very non-uniform profile in the range of $1530 \mathrm{~nm}$ $1565 \mathrm{~nm}$ for this situation, while these amounts of fluctuations are acceptable results in L-band

Received: 12 January 2016 / Revised: 3 June 2016

(C) The Author(s) 2016. This article is published with open access at Springerlink.com

DOI: $10.1007 / \mathrm{s} 13320-016-0314-\mathrm{z}$

Article type: Regular 
windows. Therefore, the SPF is not a suitable way for the C-band region. According to the experimental tests carried out by J. Yang et al., the ripple around $\pm 0.4 \mathrm{~dB}$ on the gain spectrum was achieved in $1570 \mathrm{~nm}-1610 \mathrm{~nm}$ with the using of two-stage pump configuration [4]. Also in the study conducted by M. K. Jazi et al., un-flatness gain spectrum around $1532 \mathrm{~nm}$ was still observed with double pass bidirectional ASE source [3]. However, the using of two or more pumps makes systems complex in operation that our concentration is flattening the gain profile in $1530 \mathrm{~nm}-1565 \mathrm{~nm}$ by simple way and components.

In a general classification, there are two ways for flattening gain spectrum. One is to apply changes to the material environment of erbium doped fibers, for example by doping EDF with $\mathrm{Al}$ [5] or $\mathrm{Zr}$ [6]. A hybrid configuration with zirconia-based erbium doped fiber and semiconductor optical amplifier produced the gain variation around $4 \mathrm{~dB}$ in $1530 \mathrm{~nm}-$ $1560 \mathrm{~nm}$. The second general way is to use the external filters, such as acousto-optic filter [7, 8], Mach-Zehnder interferometer [9], and fiber loop mirror $[9,10]$.

The performance of these filters is creating inverse of ASE spectrum to achieve flatness. The combination of ASE and the inverse one removes the un-flatness region, and then the gain spectrum is uniformed.

Long period fiber gratings (LPFG) that have attractive sensing applications $[11,12]$ (for example in refractive index [13, 14], strain, and temperature sensors $[14,15])$ are suitable filters because of their low insertion and return loss. These filters with different fabrication methods $[16,17]$ and implementation capabilities on several kinds of fibers (such as photonic crystal fibers [18] and the three-layer fibers [19]) are used with various techniques for equalization.

The LPFG promotes the coupling mode between the propagating light of fundamental core mode (LP01) and the co-propagating cladding modes. Because of scattering losses, the light coupled to the cladding modes decays fast in the guided core mode observed at the output end of LPFG [19, 20]. Therefore, transmission spectrum of this type of filter has a series of attenuation bands which are employed for the ripple compensation in EDF gain.

There are several gain flattening methods that have employed LPGs. At the effect of acoustic wave $[7,8]$, temperature $[21,22]$, twisting $[23,24]$, and bending process on LPGs, the fluctuations are decreased in around $30 \mathrm{~nm}$ on bandwidth by LPG length more than $51 \mathrm{~mm}$. However, we observed gain flattening around $1 \mathrm{~dB}$ due to new design of LPG on extended bandwidth by employing simple equipments.

The proposed method in this study is following the study on fabrication of ASE source by Kanani et al. [3]. Our purposed work is making a gain flattening filter for this type of source. According to the survey about ASE sources in [3], single pass backward (SPB) ASE sources is suitable for the gain flattening in $\mathrm{C}$-band telecommunication region owning to its stable broad-band spectrum and high output power rather than forward sources.

In this research, by design and fabrication of new kind of ULPFG with fat gratings and combination with another LPG on SMF-28, the flatness gain is obtained. This gain flattening filter is fabricated in the research center by point-to-point arc-discharge method which is simple and affordable.

\section{Experimental setup and results}

The performed steps for realization and fabrication arrays based on LPG as a filter are described in details.

\subsection{Designing setup}

The simulation setup is illustrated in Fig. 1 by OptiSystem software based on broad band non-coherent ASE source, LPG, optical spectrum analyser, optical isolator, and other components.

As shown in Fig. 1, the 974-nm laser diode is employed to pump the EDF with $100 \mathrm{~mW}$ pump power through the WDM as a C-band SPB ASE source. Figure 2 shows the simulated spectrum of this source in $\mathrm{C}$ band region. 


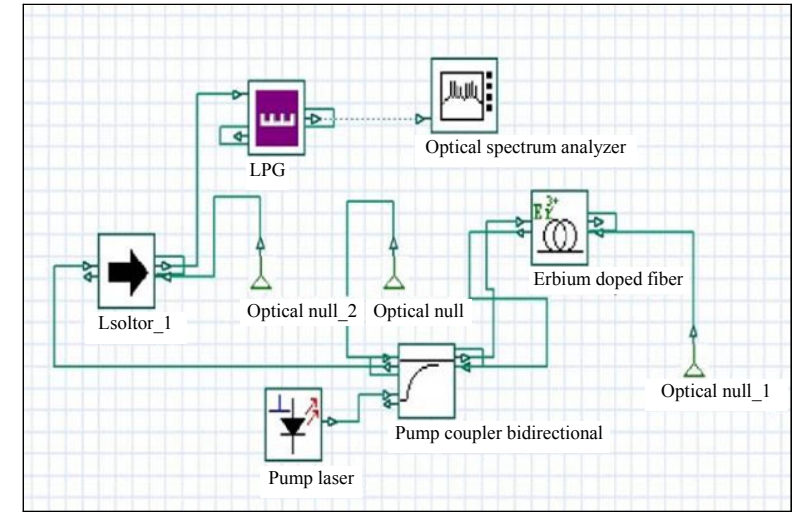

Fig. 1 Configuration of designed set-up in OptiSystem software

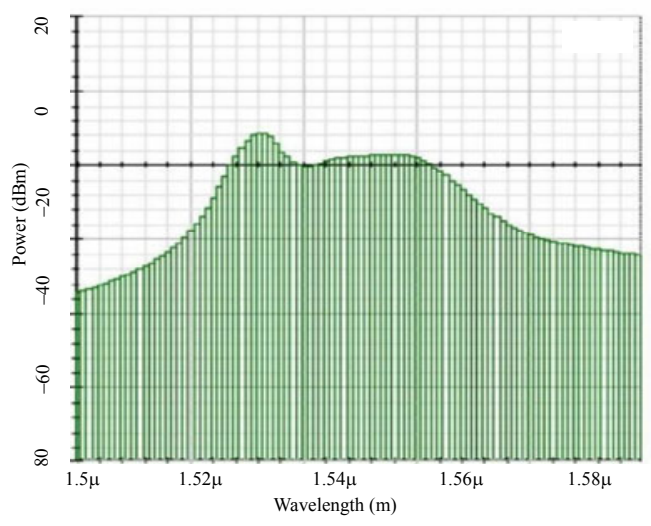

Fig. 2 Simulated spectrum of the proposed ASE source in C-band.

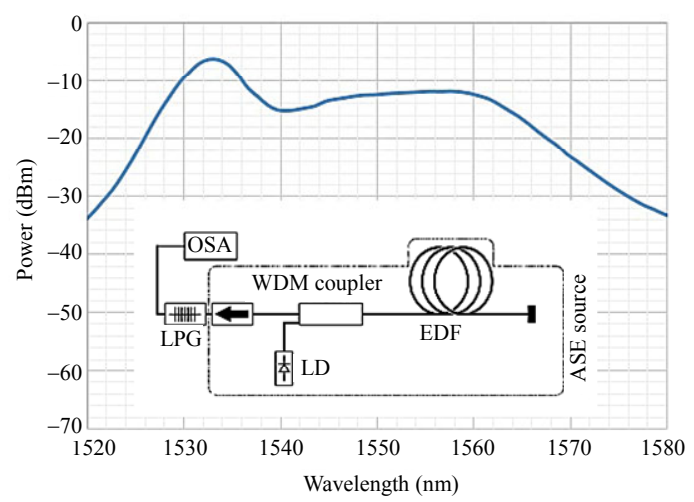

Fig. 3 Experimental spectrum of the proposed ASE source.

A minimum length of $\operatorname{EDF}(3 \mathrm{~m})$ is used as the active medium in simulation and experimental work where the fiber doping concentration is $1 / 9 \times 10^{25}$ ions $/ \mathrm{m}^{3}$ with an NA of 0.2 . The $3-\mathrm{dB}$ optical coupler or WDM coupler is placed at the end of the laser diode to transfer power to EDF (inset in Fig. 3). As a result, the variation of gain spectrum against pump power due to the experimental ASE source is shown in Fig. 3. According to this figure, a maximum peak power exists around $1532 \mathrm{~nm}$ and the fluctuations of the gain profile in $\mathrm{C}$ band interval are around $8.69 \mathrm{~dB}$.

A comparison between simulation and experimental results in Figs. 2 and 3 reveals only a little loss due to experimental conditions.

\subsection{Fabrication method of ULPFG}

According to the simple available equipment, a point-to-point arc-discharge method for ULPFG fabrication is employed in this study. Since the thickness of induced grating by arc is $959 \mu \mathrm{m}$, and distance between each grating is tuned on $630 \mu \mathrm{m}$, the period and length of the grating is obtained around $1589 \mu \mathrm{m}$ and $39 \mathrm{~mm}$, respectively. The operating wavelength is also considered as $1532 \mathrm{~nm}$.

\subsection{Performance of fabricated ULPG}

After fabricating the mentioned ULPFG in the previous step and entering the light to it, the process that transferring energy from light of fundamental mode (LP-01) into forward propagating cladding mode (LP-02) is done. Designing ULPG leads to create the loss band around $1532 \mathrm{~nm}$ and the transmission spectrum of ULPFG experiences the decrement in power in this region. When this filter is set in the following ASE setup, the decrement level of power around the peak takes place and the uniform gain profile is achieved. However, the performance of ULPFG as a gain flattening filter is shown in Fig. 4.

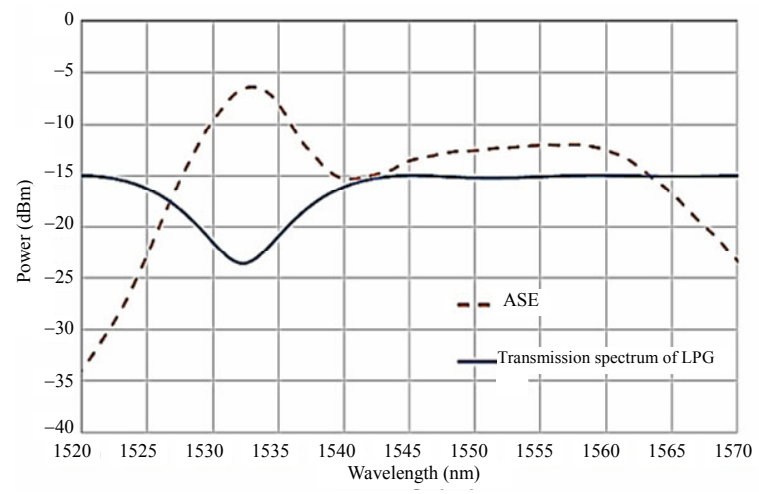

Fig. 4 Performance of ULPG for gain flattening filter. 


\subsection{Novelty in fabricating LPG}

In order to configure LPG for gain equalization, the first stage is writing ULPG with 25 segments and 39-mm length of grating on the SMF-28. The fluctuations of ASE profile decrease to $6.3 \mathrm{~dB}$ after passing from this LPG (Fig. 5). The aim of the second stage is to improve this spectrum and decrease the fluctuations greatly. So, a new kind of ULPG is designed and demonstrated in this study.

In the fabrication process of LPFGs, to implement uniform gratings, the fiber is fixed in the device, and the gratings are written on it, so that one end of the fiber is fixed, and another end is strained with a weight. In novelty process, LPFG has made with pushing two ends of the fiber towards each other while writing gratings. So the gratings are made in a fat shape (Fig. 6). However, with minimum 5 fat segments on $8 \mathrm{~mm}$ in continuing of previous ULPFG with 25 segments, more flattening gain spectrum is obtained (Fig. 5).

However, two grating series are engraved on the normal fiber. The ASE profiles of these processes are shown in Fig. 5. This figure shows the ripple of ASE spectrum that is greatly reduced by employing of two ULPFG series as a filter.

At the best final condition, by inserting of the 5 fat gratings, the fluctuations around $3.4 \mathrm{~dB}$ are obtained.

As mentioned before, Fig. 6 shows the designing model of this new type of ULPFG with fat gratings that have higher refractive index rather than any region of the fiber.

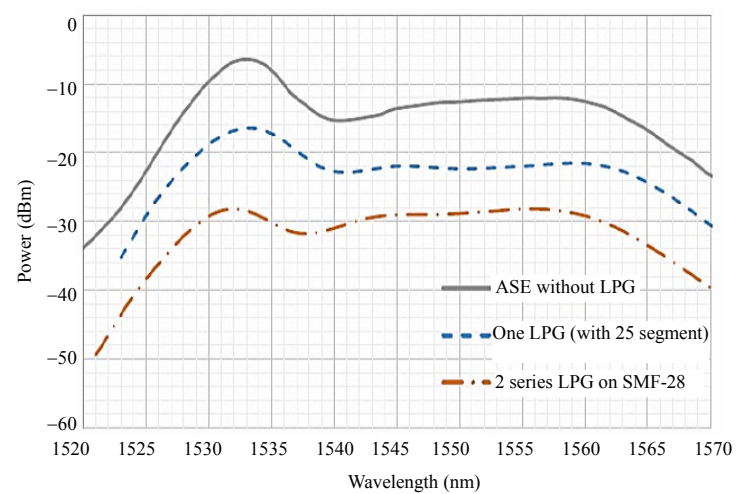

Fig. 5 ASE spectrum in absence of any filter (solid curve), ASE after passing from one ULPG with 25 segment (dash line curve), and ASE after two ULPFG (dash-dot curve).

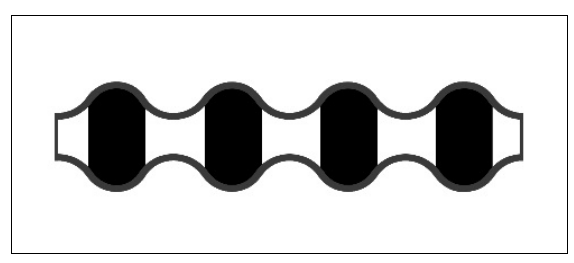

Fig. 6 Novel LPG design for gain flattening with fat grating.

\subsection{ASE spectrum with different input powers}

Finally, in order to achieve the compact filter, two kinds of ULPG are fabricated on the fiber. The gain profile is compared in Fig.7, by prepared filter and the source in different pump powers of $100 \mathrm{~mW}$ and $300 \mathrm{~mW}$, respectively, the gain profile is compared in Fig. 7. As shown in this figure, the fluctuations of pump power in $100 \mathrm{~mW}$ are less than that in $300 \mathrm{~mW}$. The ripple rate for $100 \mathrm{~mW}$ is around $3.4 \mathrm{dBm}$ while for $300 \mathrm{~mW}$ is around $4.5 \mathrm{dBm}$. So, for the less power in this study, the gain profile has become more flatten.

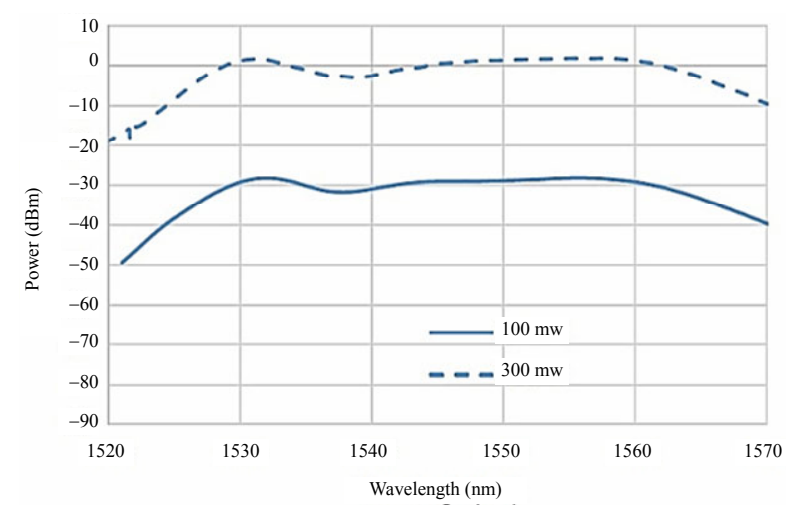

Fig. 7 Comparison of the flattened gain profile in $100 \mathrm{~mW}$ and $300 \mathrm{~mW}$ pump power output.

\section{Comparison of results}

Table 1 shows the comparison between results and selection of the best filter for the equalization of ASE profile in this study.

Table 1 Comparing of the fluctuations in gain profile.

\begin{tabular}{ccc}
\hline Kind of profile & $\begin{array}{c}\text { Value of } \\
\text { fluctuation }(\mathrm{dB})\end{array}$ & $\begin{array}{c}\text { Optimum } \\
\text { state }\end{array}$ \\
\hline ASE without filter & 8.69 & \\
ASE+ ULPFG (25 segment) & 6.3 & \\
ASE+ ULPFG (25 segment)+ & $3.4( \pm 1.7)$ & $\checkmark$ \\
ULPFG (5 fat segment) & 3.3 & \\
\hline
\end{tabular}

Compared with other methods for equalization gain profile, the following points should be considered: 
(1) The minimum length of erbium doped fiber that used (around $3 \mathrm{~m}$ in compare to other reports $>10 \mathrm{~m}$ ) is so better because of compacting integrated circuits and economic issue.

(2) The length of LPFG that is fabricated is $39 \mathrm{~mm}$ while in many studies it is longer.

(3) The important point is a simple fabrication method that is achieved by arc-discharge system and simple equipments. Although, some of investigations have better fluctuations in ASE spectrum, the results with this method are more desirable rather than the complicated fabrication way.

\section{Conclusions}

In summary, a novel filter was proposed for flattening the gain spectrum of ASE in this research. The SPB ASE source was used and gain flattening profile with employing two stage ULPG was achieved, where the second ULPFG was made of fat gratings. In this way, the input power was $100 \mathrm{~mW}$. With this low level of pump power, the uniform gain profile in $\mathrm{C}$ band region with $3.4( \pm 1.7) \mathrm{dB}$ ripple rate was observed.

Hence, the recombination of two LPGs by using the novel ULPG with fat grating at the comparison with other methods is easy, compact, and affordable for employing in various applications. The ASE flat spectrum is also very applicable for broadband sensors, WDM systems, filters, and so on.

\section{References}

[1] K. Toge and F. Ito, "Recent research and development of optical fiber monitoring in communication systems," Photonic Sensors, 2013, 3(4): 304-313.

[2] J. Tiana, Y. Yaoa, Y. X. Suna, X. C. Xub, X. H. Zhaoa, and D. Y. Chen, "Flat broadband erbium-doped fiber ASE source based on symmetric nonlinear optical loop mirror," Laser Physics, 2010, 20(8): 1760-1766.

[3] M. K. Jazi, S. Shahi, M. J. Hekmat, H. Saghafifar, A. T. Khuzani, H. Khalilian, et al., "The evaluation of various designs upon C\&L band super fluorescent sources based erbium doped fiber," Laser Physics, 2013, 23(6): 553-559.
[4] J. Yang, X. Meng, C. Liu, and C. Liu, "Gain-flattened two-stage L-band erbium-doped fiber amplifier by weak gain-clamped technique," Optical Engineering, 2015, 54(3): 036107-1-036107-5.

[5] H. Lin, W. Fan, and W. Han, "Broad gain of the Er/Al- doped fiber amplifier by pumping with a white light-emitting diode," Journal of Luminescence, 2014 146(1): 87-90.

[6] N. A. D. Huri, A. Hamzah, H. Arof, H. Ahmad, and S. W. Harun, "Hybrid flat gain c-band optical amplifier with zr-based erbium-doped fiber and semiconductor optical amplifier," Laser Physics, 2011, 21(1): 202-204.

[7] C. A. F. Marques, R. A. Oliveira, A. A. P. Pohl, and R. N. Nogueira, "Adjustable EDFA gain equalization filter based on a single LPG excited by flexural acoustic waves for future DWDM networks," in International Conference on Fibre Optics and Photonics, 2012, 180: 3770-3774.

[8] A. A. P. Pohl, R. A. Oliveira, R. E. D. Silva, C. A. F. Marques, P. D. T. Neves Jr, K. Cook, et al., "Advances and new applications using the acousto-optic effect in optical fibers," Photonic Sensors, 2013, 3(1): 1-25,

[9] X. Xue, W. Zhang, L. Yin, S. Wei, Sh. Gao, P. Geng, et al., "All-fiber intermodal Mach-Zehnder interferometer based on a long-period fiber grating combined with a fiber bitaper," Optics Communications, 2012, 285: 3935-3938.

[10] N. Kumar and K. Ramachandran, "Mach-Zehnder interferometer concatenated fiber loop mirror based gain equalization filter for an EDFA," Optics Communications, 2013, 289(4): 92-96.

[11] A. P. Zhang, S. Gao, G. Yan, and Y. Bai, "Advances in optical fiber Bragg grating sensor technologies," Photonic Sensors, 2012, 2(1): 1-13.

[12] K. Kalli, T. Allsop, K. Zhou, G. Smith, M. Komodromos, D. Webb, et al., "Sensing properties of femtosecond laser-inscribed long period gratings in photonic crystal fiber," Photonic Sensors, 2011, 1(3): 228-233.

[13] A. Singh, "Long period fiber grating based refractive index sensor with enhanced sensitivity using michelson interferometric arrangement," Photonic Sensors, 2015, 5(2): 172-179.

[14] Q. Huang, Y. Yu, Z. Ou, X. Chen, J. Wang, P. Yan, et al., "Refractive index and strain sensitivities of a long period fiber grating," Photonic Sensors, 2014, 4(1): 92-96.

[15] R. C. Chaves, A. D. A. P. Pohl, I. Abe, R. Sebem, and A. Paterno, "Strain and temperature characterization of LPGs written by $\mathrm{CO} 2$ laser in pure silica LMA photonic crystal fibers," Photonic 
Sensors, 2015, 5(3): 241-250.

[16] X. Xu, J. Tang, J. Zhao, K. Yang, C. Fu, Q. Wang, et al., "Post-treatment techniques for enhancing mode-coupling in long period fiber gratings induced by CO2 laser," Photonic Sensors, 2015, 5(4): 339-344.

[17] M. Melo and P. V. S. Marques, "Fabrication of tailored Bragg gratings by the phase mask dithering/moving technique," Photonic Sensors, 2013, 3(1): 81-96.

[18] J. Ju and W. Jin, "Long period gratings in photonic crystal fibers," Photonic Sensors, 2012, 2(1): 65-70.

[19] A. Singh, "Study of modeling aspects of long period fiber grating using three-layer fiber geometry," Photonic Sensors, 2015, 5(1): 32-42.

[20] Q. Li, F. Yan, P. Liu, W. Peng, G. Yin, and T. Feng, "Analysis of transmission characteristics of tilted long period fiber gratings with full vector complex coupled mode theory," Photonic Sensors, 2012, 2(2):
$158-165$.

[21] J. K. Bae, J. Bae, S. H. Kim, N. Park, and S. B. Lee, "Dynamic EDFA gain-flattening filter using two lpfgs with divided coil heaters," IEEE Photonics Technology Letters, 2005, 17(6): 1226-1228.

[22] V. Nascimento, J. D. Oliveira, V. B. Ribeiro, and A. C. Borndonalli, "Dynamic EDFA gain spectrum equalizer using temperature controlled optoceramic filter array," in IEEE Microwave \& Optoelectronics Conference (IMOC), Natal, 2011, pp. 273-276.

[23] R. B. Shang, W. G. Zhang, W. B. Zhu, P. C. Geng, J. Ruan, S. C. Gao, et al., "Fabrication of twisted long period fiber gratings with high frequency $\mathrm{CO} 2$ laser pulses and its bend sensing," Journal of Optics, 2013, 15(7): 75402-75407.

[24] W. Jin and H. Xuan, "Rocking long period gratings in single mode fibers," IEEE Lightwave Technology, 2013, 31(18): 3117-3122. 